\title{
Turbulent Output-Based Anisotropic Adaptation
}

\author{
Michael A. Park* \\ Jan-Reneé Carlson* \\ NASA Langley Research Center, Hampton, VA 23681
}

\begin{abstract}
Controlling discretization error is a remaining challenge for computational fluid dynamics simulation. Grid adaptation is applied to reduce estimated discretization error in drag or pressure integral output functions. To enable application to high $\mathcal{O}\left(10^{7}\right)$ Reynolds number turbulent flows, a hybrid approach is utilized that freezes the near-wall boundary layer grids and adapts the grid away from the no slip boundaries. The hybrid approach is not applicable to problems with under resolved initial boundary layer grids, but is a powerful technique for problems with important off-body anisotropic features. Supersonic nozzle plume, turbulent flat plate, and shock-boundary layer interaction examples are presented with comparisons to experimental measurements of pressure and velocity. Adapted grids are produced that resolve off-body features in locations that are not known a priori.
\end{abstract}

\section{Introduction}

Obtaining a suitable grid remains one of the most difficult parts of the entire Computational Fluid Dynamics (CFD) simulation process. Grids must resolve flow features to provide adequate control of discretization error and remain small enough to permit reasonable computation times. The AIAA Drag Prediction Workshops $^{1}$ are well-documented examples of the difficulty of suitable grid generation even under the watchful eyes of experts. Mavriplis ${ }^{2}$ shows that discretization error still dominates other error sources (e.g., turbulence model distance function calculation, thin-layer viscous approximation, level of artificial dissipation) even with extremely fine grids that are computable with today's supercomputers. Chaffin and Pirzadeh ${ }^{3}$ detail the effort required to manually specify grid resolution for accurate three dimensional (3D) high-lift computations. The high degree of manual intervention required in these cases prohibits the use of automated design tools. The manual interaction required to create a suitable grid is reduced with adaptive grid methods. Automated parameter studies ${ }^{4}$ and design ${ }^{5}$ are possible with an automated grid generation and adaptation process. Accurate final adapted solutions can be produced that are independent of coarse initial grids. ${ }^{6-9}$ There are two main barriers to the use of grid adaptation in CFD simulation. The first is the ability of the adaptive indicator to produce an improved grid that will eventually yield a solution suitable for engineering application. The second is the robustness of grid adaptation mechanics.

Adaptive grid methods are designed to specify a resolution request that equidistributes and minimizes error estimates. The improved resolution request is commonly based on local error estimates. ${ }^{10-13}$ Uniformly reducing the errors associated with all local-error sources of the flow may not be optimal from an engineering context, where calculating an output functional may be of greater concern. An alternative method is to estimate the error in the calculation of a specified engineering output functional. ${ }^{14-17}$ Output error indicators utilize the dual or adjoint solution of an output functional to account for the impact of local error as well as the transport of these local errors throughout the problem domain to improve the calculation of that output functional. This output-adaptive approach has been applied to finite-volume simulations in two dimensions $(2 \mathrm{D})^{16}$ and $3 \mathrm{D}^{18}$ with a parallel execution scheme. ${ }^{19}$

Adapting 3D grids in the neighborhood of curved boundaries to an anisotropic metric is a difficult problem. Most of the difficulty lies in simultaneously maintaining the boundary nodes on the geometry description and element validity. The projection of nodes to curved geometry has been applied as a postprocessing step after surface refinement to a linear surface approximation. Directly moving the surface nodes

${ }^{*}$ Research Scientist, Computational AeroSciences Branch, NASA Langley Research Center, MS 128, Hampton, VA 23681, AIAA Senior Member. 
to the projected location without modification of the volume grid may result in invalid, inverted elements. The displacements required to project surface nodes can be distributed throughout the grid to prevent the invalidation of elements near surfaces. The volume displacement has been computed via Laplacian smoothing, linear springs, torsional springs, trusses, and linear elasticity. While these methods reduce the incidence of invalid element creation, they are not guaranteed to prevent invalid elements while projecting boundary nodes. Iterative methods have been developed to project the surface node to the "first problem plane" ${ }^{20,21}$ (past the first element that becomes inverted during projection) with various combinations of edge swapping and node movement. However, grid regeneration ${ }^{22}$ is reported to be necessary when this iterative method fails.

This study avoids the issue of adapting high aspect ratio elements on curved boundaries by utilizing a hybrid approach which freezes the near-wall grid and adapts elements outside this zone. This simplifies the adaptation mechanics and increases adaptation robustness. This hybrid approach is not applicable to problems with under resolved initial boundary layer grids, because that portion of the grid is held constant during the simulation. However, the hybrid approach is a powerful technique for problems with important off-body anisotropic features, i.e., plumes, sonic boom propagation, and bow shocks. This study is an extension of 3D output-based adaptation to subsonic and supersonic turbulent flows.

\section{Flow and Adjoint Solver}

Fully Unstructured Navier-Stokes Three-Dimensional (FUN3D) is a suite of codes for finite-volume CFD. ${ }^{23,24}$ The FUN3D website, http://fun3d.larc.nasa.gov, contains the user manual and an extensive list of references. FUN3D is able to solve incompressible, Euler, and Reynolds-averaged Navier-Stokes (RANS) flow equations, either tightly or loosely coupled to a turbulence model. Domain decomposition is employed to fully exploit the distributed architectures predominate in high-performance computing to increase problem size and reduce the execution time of the simulation process. The compressible flow solver employs an unstructured finite-volume method for conserved variables stored at each node in the grid,

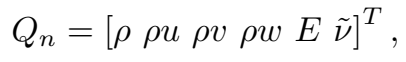

where $\rho$ is density, $u, v$, and $w$ are velocity, and $E$ is total energy per unit volume. The Spalart-Allmaras ${ }^{25,26}$ (S-A) turbulence model quantity $\tilde{\nu}$ is also included. FUN3D can utilize general mixed element grids, but only tetrahedral grids are used in this study. All node-based variables in the grid $Q$ are computed by driving the flow equation residual $R$ to steady-state with an implicit point-iterative method. The present study employs the RANS equations coupled to the S-A turbulence model.

After the steady-state flow solution is obtained, the discrete adjoint equations ${ }^{27,28}$ are solved to complete the dual problem. To derive these equations, the residual $R$ is augmented with a source term $S$ and the sensitivity of the output function with respect to this source term is sought. A potential application of this sensitivity is to model truncation error as a source term and determine the effect of this error on the output function $f$. A Lagrangian is formed from $f$ with $R$ included as a constraint,

$$
\mathcal{L}(Q, S, \lambda)=f(Q, S)+\lambda^{T} R(Q, S) .
$$

Each adjoint variable $\lambda \in \mathcal{R}^{6 N}$ corresponds to a flow equation. The Lagrangian is differentiated with respect to $S$,

$$
\frac{\partial \mathcal{L}}{\partial S}=\frac{\partial f}{\partial S}+\left(\frac{\partial Q}{\partial S}\right)^{T}\left(\frac{\partial f}{\partial Q}+\lambda^{T} \frac{\partial R}{\partial Q}\right)+\lambda^{T} \frac{\partial R}{\partial S} .
$$

The dependency on $\frac{\partial Q}{\partial S}$ can be eliminated by solving the adjoint equation,

$$
\left(\frac{\partial R}{\partial Q}\right)^{T} \lambda=-\left(\frac{\partial f}{\partial Q}\right)^{T}
$$

With the adjoint equation satisfied, the sensitivity of the output function can be expressed as,

$$
\frac{\partial \mathcal{L}}{\partial S}=\frac{\partial f}{\partial S}+\lambda^{T} \frac{\partial R}{\partial S} .
$$

The linear adjoint equations in Eq. (4) are solved with a dual-consistent time-marching method. ${ }^{29,30}$ The iterative method is constructed such that an adjoint solution exactly dual to a direct differentiation 
approach is recovered at each time step, yielding a convergence rate which is asymptotically equivalent to that of the flow equations.

\section{Output-Based Adaptation}

Venditti ${ }^{16}$ describes an output-based error estimation and adaptation scheme. This has been implemented in $3 \mathrm{D}^{18}$ with a parallel ${ }^{19}$ implementation. The adaptation intensity $I$ is formed on an embedded grid,

$$
I_{n}^{e}=\frac{\left|\left(\lambda^{H}-\lambda^{L}\right)^{T} R\left(Q^{H}\right)\right|+\left|R_{\lambda}\left(\lambda^{H}\right)\left(Q^{H}-Q^{L}\right)^{T}\right|}{2},
$$

where $\lambda^{H}$ and $\lambda^{L}$ are the adjoint solution and $Q^{H}$ and $Q^{L}$ are the flow solution interpolated to the embedded grid. The ()$^{H}$ interpolation is based on a quadratic fit of the original grid solution and its reconstructed gradients. The ()$^{L}$ interpolation is linear. The flow $Q$ and adjoint $R_{\lambda}$ residual operators are also on the embedded grid. The embedded grid $I_{n}^{e}$ is restricted to the original grid $I_{n}$. The embedded grid required for the Venditti error estimator can be prohibitively large (eight times original grid) for large 3D problems, which effectively limits the problem size of the output adaptive method. A single grid error estimation procedure is under development. It has been applied to Euler problems, ${ }^{31}$ but not evaluated for turbulent simulations.

Venditti ${ }^{16}$ provides a procedure to calculate a new grid spacing request from the adaptive indicator $I_{n}$ and an error tolerance $\operatorname{tol}_{\Omega}$. The adaptation indicator is summed to find the global indicator $I_{\Omega}=\sum I_{n}$. The ratio of the remaining error to a user-specified error tolerance tol $\Omega$ is

$$
\epsilon_{\Omega}=\frac{I_{\Omega}}{\operatorname{tol}_{\Omega}}
$$

The ratio of the control volume indicator to an equal share of $\operatorname{tol}_{\Omega}$ is

$$
\epsilon_{n}=\frac{N}{\operatorname{tol}_{\Omega}} I_{n}
$$

where $N$ is the number of control volumes. When a cost function does not have an intuitive error tolerance, i.e., sonic boom surface pressure integrals, the tol $\mathrm{l}_{\Omega}$ is set to half $I_{\Omega}$ at each adaptive iteration. The requested isotropic element length $h$ is computed with an estimate of the spacing on the original mesh $h^{0}$ and the global and local error ratios,

$$
h_{n}=h_{n}^{0}\left(\frac{1}{\epsilon_{\Omega} \epsilon_{n}}\right)^{\omega}
$$

where an exponent of $\omega=0.20$ is based on an a priori estimate of the spatial error convergence. The anisotropy of mesh elements is based on the the Mach Hessian, where the element size in the smallest spacing direction is dictated by the adjoint adaptation parameter.

A metric tensor $M$ is employed to define the desired multidimensional grid resolution because it is a natural way to express local interpolation error estimates of linear functions. ${ }^{10,32-34}$ The symmetric positive definite matrix $M$ in $3 \mathrm{D}$ has the diagonal decomposition

$$
M=X\left[\begin{array}{lll}
\Lambda_{1} & & \\
& \Lambda_{2} & \\
& & \Lambda_{3}
\end{array}\right] X^{T}=X \Lambda X^{T} .
$$

The eigenvectors $X$ define an orthonormal basis with length specifications $h_{i}$ in this basis,

$$
\Lambda=\left[\begin{array}{ccc}
\left(\frac{1}{h_{1}}\right)^{2} & & \\
& \left(\frac{1}{h_{2}}\right)^{2} & \\
& & \left(\frac{1}{h_{3}}\right)^{2}
\end{array}\right] .
$$

This metric can be interpreted as an ellipsoid with major and minor axes of directions $X_{i}$ and lengths $h_{i}{ }^{10}$ 
To form the metric $M$, the Mach Hessian $H$ is decomposed into eigenvalues $\Lambda$ and eigenvectors $X$. The matrix $M$ has the same eigenvectors as matrix $H$ to keep the same principle directions and absolute value of the corresponding eigenvalues to make it symmetric positive definite. The adjoint adaptation parameter is incorporated into the Hessian framework by scaling the three eigenvalues so that the largest eigenvalue corresponds to the adjoint adaptation spacing requirement. The eigenvalues and corresponding eigenvectors are sorted so that $\left|\Lambda_{1}\right|>\left|\Lambda_{2}\right|>\left|\Lambda_{3}\right|$. Thus, after scaling, the largest eigenvalue is

$$
\Lambda_{1}=\left(\frac{1}{h}\right)^{2}
$$

where the specified element length $h$ is computed by the output error estimation process, Eq. (9).

Parallel grid mechanics described by Park and Darmofal ${ }^{31,35}$ are used to modify the tetrahedral background grid. The local elemental operators of node insertion, node movement, element swap, and element collapse are utilized to iteratively drive the edge lengths to unity in $M$.

\section{Jet Plume Simulation}

The supersonic wind tunnel geometry is shown in Fig. 1. This case is chosen because it has a complex shock and expansion structures in locations that are not known a priori. These shocks and expansions also interact with viscous boundary and sheer layers in a way that makes manual specification of grid element size and stretching difficult even if the locations of these features are known. The nozzle 6 from Putnam and Capone ${ }^{36}$ has a exit-to-throat area ratio of 1.722 and a boattail angle of 5 deg. The ratio of jet total pressure to free stream static pressure is 8.12. The computational domain is a quarter of the axisymmetric model and the blade sting is ignored. The free stream Mach number is 2.2. The Reynolds number (based on maximum nozzle diameter $D=15.24 \mathrm{~cm}$ ) is $1.86 \times 10^{6}$. The $\mathrm{S}$-A turbulence model is utilized. This particular turbulence model is not ideal for modeling the shear flows of the plume, but has the advantage of a verified adjoint implementation in FUN3D. The computation domain for the nozzle plenum begins at the screens of the wind tunnel model where a total pressure boundary condition is applied. ${ }^{37}$ The outflow boundary state is extrapolated from the interior of the domain. Free stream boundary conditions are applied to the far-field boundaries. The symmetry planes of the configuration are treated with $y$ - and $z$-symmetry.

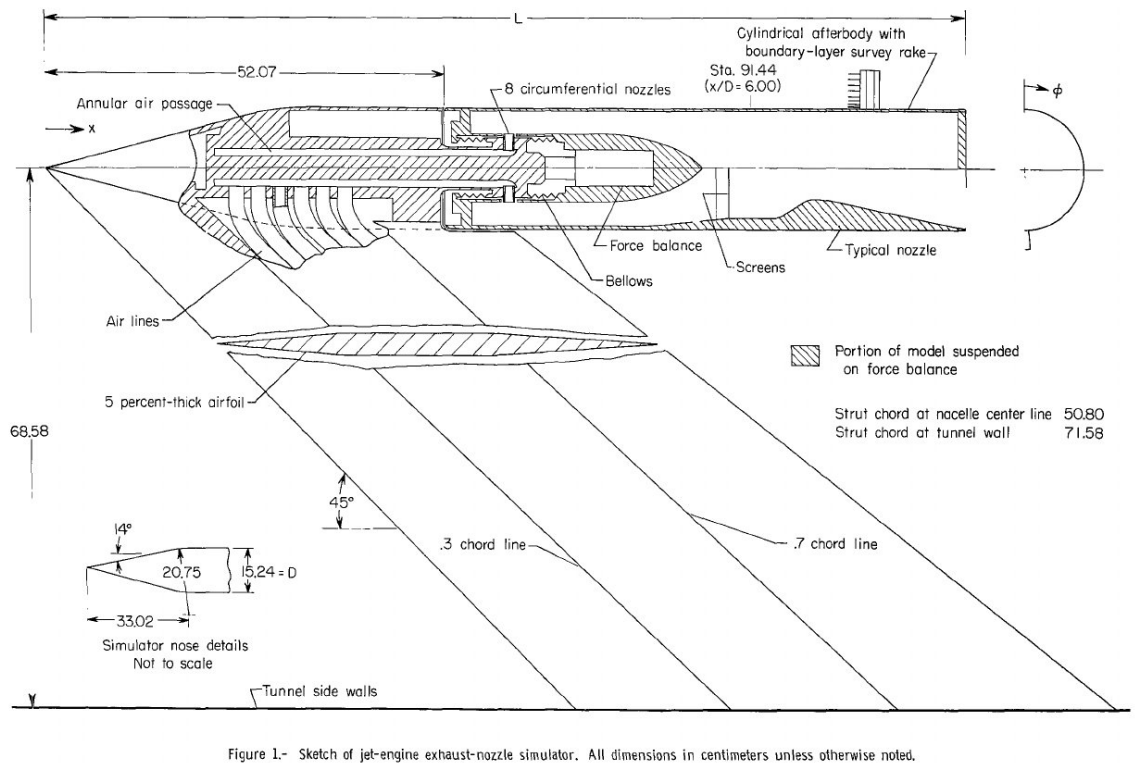

Figure 1. Plume test geometry. ${ }^{36}$

Accurate predictions of near-field pressure signatures are obtained with the output-based adaptation algorithm, which reduces estimated error in an off-body pressure integral. The integral of quadratic pressure 
deviation over a surface $s$ in the domain is

$$
f=\frac{1}{A_{s}} \iint_{s}\left(\frac{p-p_{\infty}}{p_{\infty}}\right)^{2} \mathrm{~d} s
$$

where $A_{s}$ is the area of the integration surface. This surface is a cylinder with radius of $D$ where the pressure field was measured in the experiment. This focuses the adaptation on improving the calculation of pressure deviation from free stream near this surface. The original grid was generated with VGrid. ${ }^{38}$ The problem was executed on 128 processors. The symmetry plane of the 3D grid is shown in Fig. 2. The original grid has 521,198 nodes and the adapted grid has 4,754,224 nodes. FUN3D utilizes a node-based algorithm, so the number of nodes is the number of control volumes. The boundary layer grid is frozen within $0.05 \mathrm{~cm}$ (0.00328 nozzle diameters) of the body, which is well inside of the boundary layer and advancing layer grid topology.

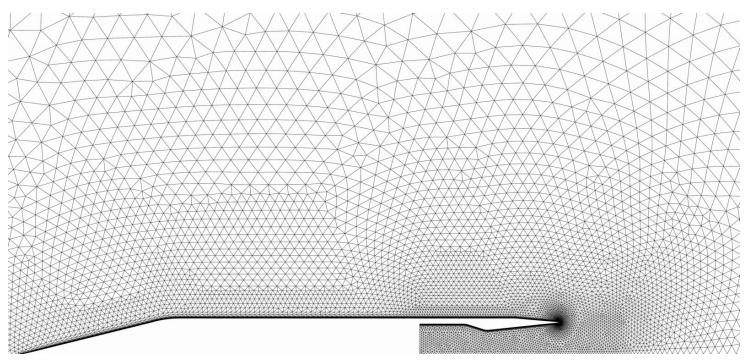

(a) Original grid.

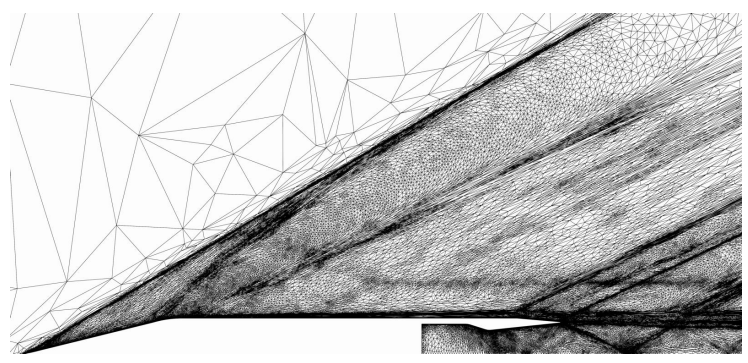

(b) Final adapted grid.

Figure 2. Symmetry plane grid.

Pressure on the original and adapted symmetry plane grids is shown in Fig. 3. The nose shock and expansion are better defined and propagated a longer distance from the body as a result of the grid adaptation. The shock train in the plume is also better defined. This is particularly evident on the aft shock where the original grid began to coarsen. Details of the plume region are shown in Fig. 4 and Fig. 5. The adapted grid is aligned with the boundary layer and shear layer. It is also aligned to the shocks and expansions. The regions where shocks or expansions interact with the boundary layer no longer have preferred alignment direction and result in isotropic refinement. The pressure field becomes more refined as a result of the adaptation, see Fig. 5 .

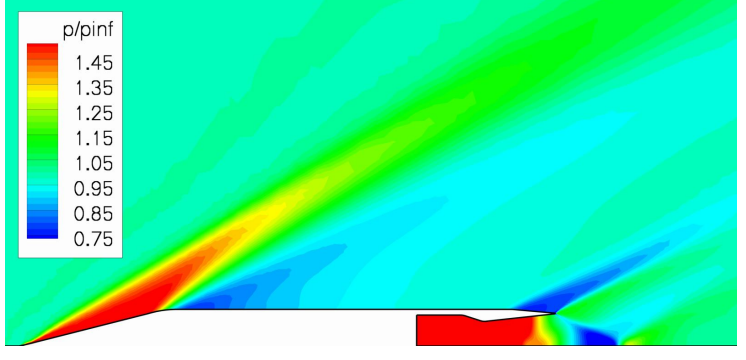

(a) Original grid.

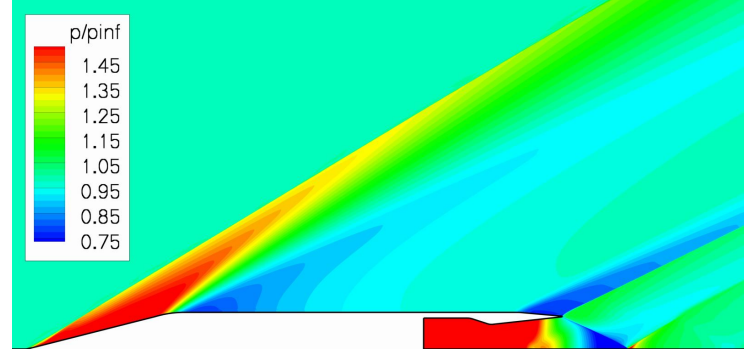

(b) Final adapted grid.

Figure 3. Pressure contours on the symmetry plane.

The pressure signature is extracted along a line that is one nozzle diameter above the configuration center line, as shown in Fig. 6. The pressure signature on the final adapted grid is the solid line. The wind tunnel data is from Putnam and Capone ${ }^{36}$ and contains measurement by two different probes. Previous computational results by Castner ${ }^{39}$ are shown as the dotted line. There is significant scatter in the experimental measurements, which may be due to irregularities on the tunnel walls or the blade sting. The pressure is not freestream ahead of the bow shock, which may indicate an offset in the experimental reference pressure. Castner did not model the fore body so the dotted line is constant forward of the boattail $x / D=7.6$. The signature due to the boattail and jet plume is shown in Fig. 7. The scatter in the wind tunnel measurements is greatest forward of the boattail expansion. The current results (solid line) accurately predict the location of the boattail expansion and primary plume shock. The predicted pressure is slightly greater than the measurements in the boattail expansion. 


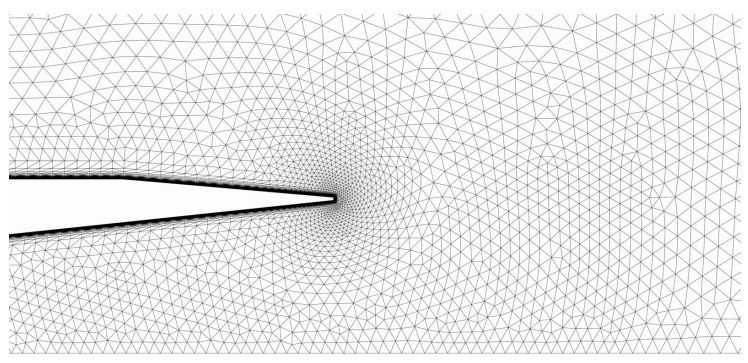

(a) Original grid.

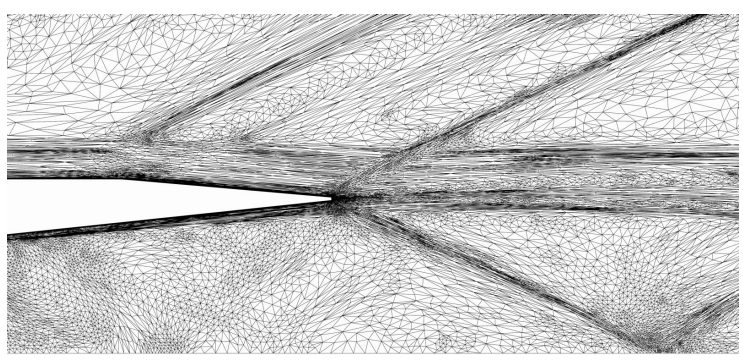

(b) Final adapted grid.

Figure 4. Symmetry plane grid nozzle exit detail.

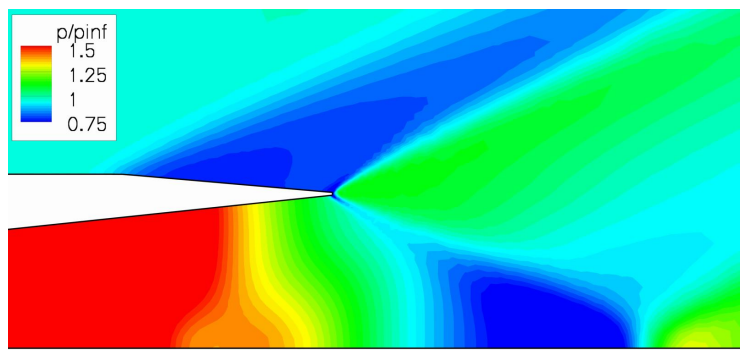

(a) Original grid.

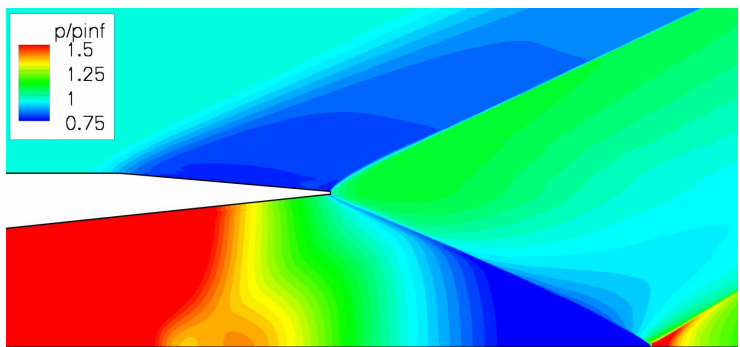

(b) Final adapted grid.

Figure 5. Nozzle exit detail of pressure contours on the symmetry plane.

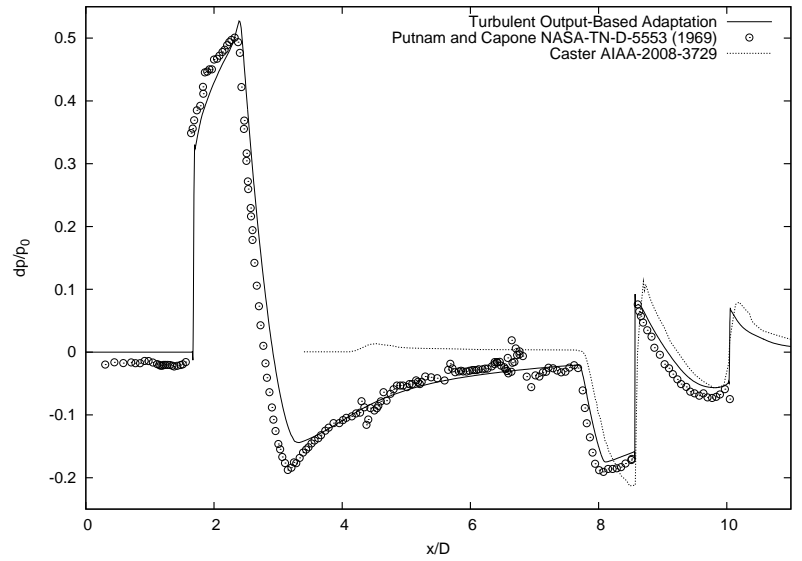

Figure 6. Entire pressure signature at a radius of one nozzle diameter above the center line.

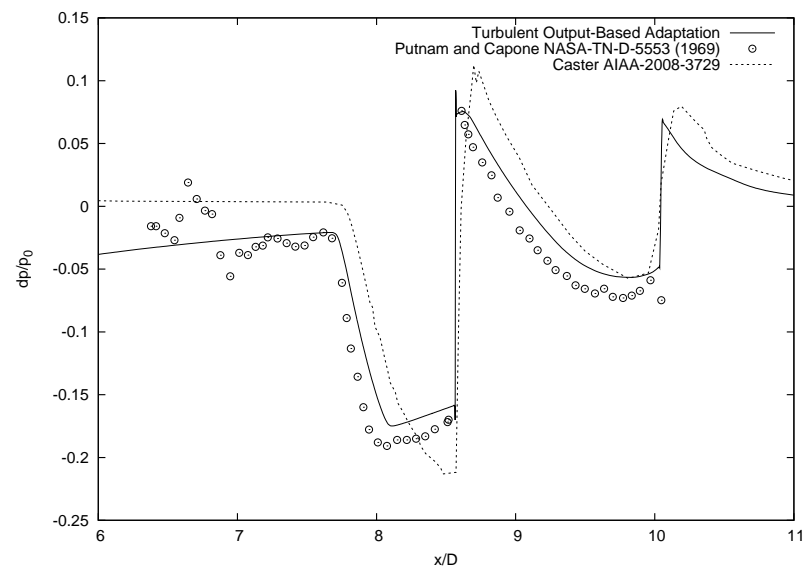

Figure 7. Aft pressure signature at a radius of one nozzle diameter above the center line. 
Putnam and Capone ${ }^{36}$ provides total pressure boundary layer profiles at a number of circumferential locations $\phi$, where $\phi=0$ is opposite the blade sting. These experimental boundary layer profiles were measured with zero boattail angle at the start of boattail for the simulated configuration. The measurements include a small range of Reynolds number between 1.55 and 2.06 million based on maximum nozzle diameter. The Reynolds number based on this $x / D=6.833$ model station is 12.69 million. A comparison of the total pressure boundary layer profiles for the original and adapted grids is provided in Fig. 8. The total pressure of the supersonic portions of the CFD profile has been reduce by using the normal shock relations, because it is assumed that the flow would experience a normal shock at the leading edge of the boundary layer probe. The wind tunnel measurements from the wind tunnel report are provided at multiple $\phi=0,45$, and 90 to give an indication of the experimental uncertainty. The blade sting is located at $\phi=180$ so the disturbed profile data at $\phi=135$ and 180 are omitted. The adapted grid is frozen below 0.00328 nozzle diameters, which is well inside the boundary layer. The outer portion of the boundary layer is under resolved in the initial grid, resulting in thicker boundary layer than the wind tunnel measurements. The adapted grid predicts a boundary layer with approximately the same thickness as the wind tunnel measurements. The adapted grid CFD predicts higher total pressure recovery for $y / D<0.05$. The turbulent velocity profile for a subsonic flat plate with adapted grid is examined in the next section to further investigate this over prediction.

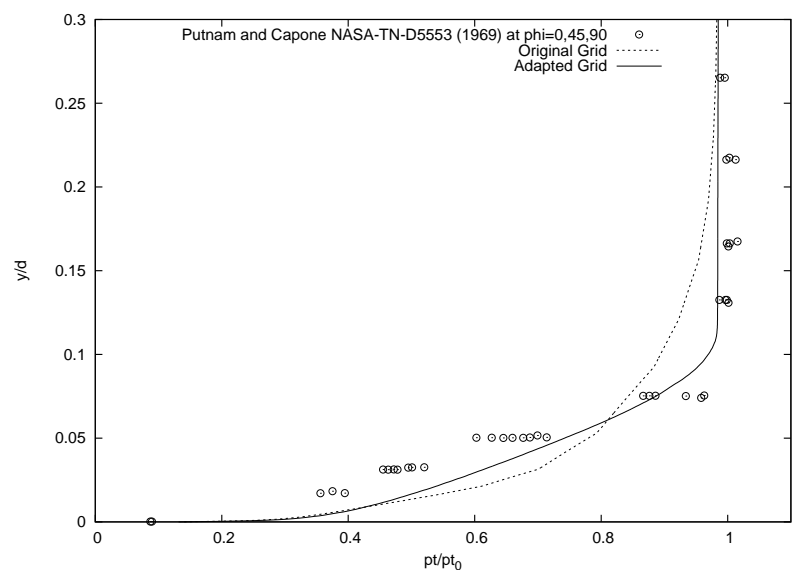

Figure 8. Total pressure profile at $x=104 \mathrm{~cm}$.

An existing inviscid output-adaptive cut-cell technique ${ }^{31,40}$ is also applied to the configuration to give an indication of the viscous effects on the signature. This comparison is included because Euler methods are the primary tool for studying near-field sonic boom signatures. The primary plume shock location is further aft for the inviscid simulation, Fig. 9. The inviscid secondary shock is further forward. The turbulent calculation better predicts the location of the primary plume shock location.

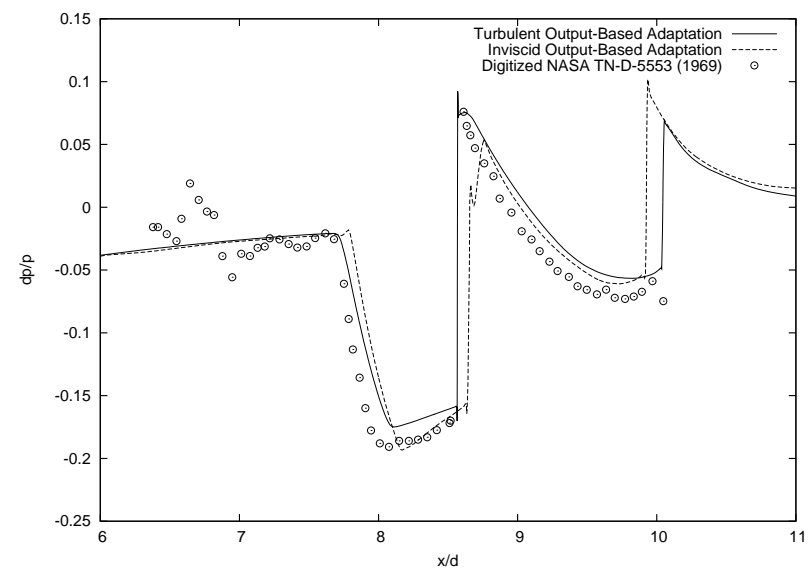

Figure 9. Aft pressure signature at a radius of one nozzle diameter above the center line. 


\section{Turbulent Flat Plate}

A turbulent flat plate is simulated with the hybrid adaptive scheme to verify the accuracy of the technique for boundary-layer flows. The selection of this case is motivated by the over prediction of total pressure (as compared to wind tunnel measurements) for the boundary layer profile in Fig. 8. Skin friction drag on the plate is used as the output to drive adaptation. The problem was executed on 64 processors. Similar conditions were investigated by Andren and Park. ${ }^{41}$ A 3D channel with a width of 0.1 is simulated to exercise the 3D adaptation mechanics. Symmetry boundary conditions are applied to both side walls of the channel. The flat plate has a length of two and the symmetry boundary condition is applied to the floor of the channel for a length of one upstream of the flat plate. The Mach number is 0.1 and the Reynolds number is $12,693,200$. The initial cell height is $1.92 \times 10^{-6}$ with a 1.1 geometric growth rate and the grid is frozen below $4.8 \times 10^{-4}$, which is equivalent to $y^{+}=212$. These conditions are chosen to correspond to the state of the boundary layer and grid of the plume case at the location that the velocity profile was extracted. The initial grid has 88,389 nodes and the adapted grid has 349,152 nodes. The original grid (constructed by subdividing structured grid hexahedra in to tetrahedra) and adapted grid are shown in Fig. 10.

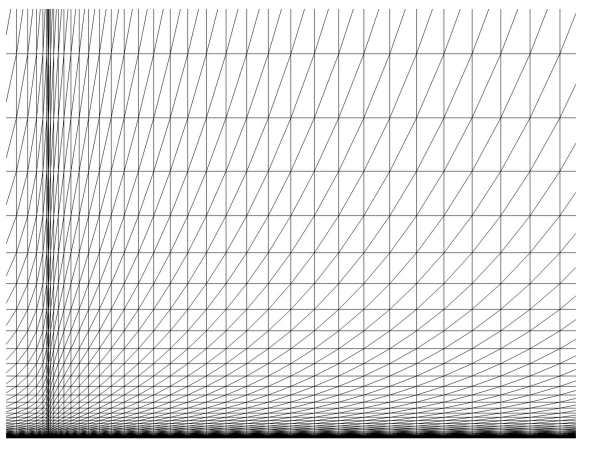

(a) Original grid.

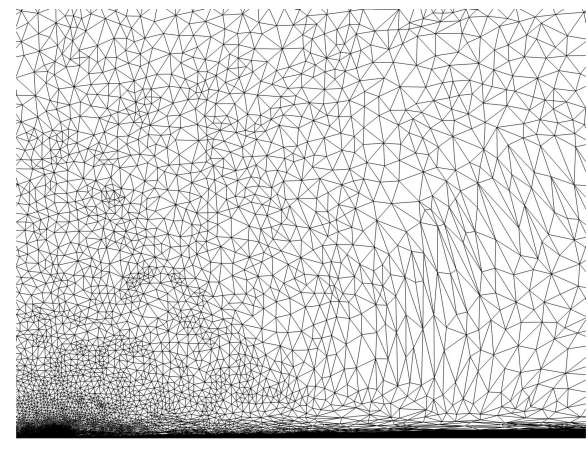

(b) Final adapted grid.

Figure 10. Symmetry plane grid for turbulent flat plate.

The velocity profile is extracted at the center of the plate, a distance of one from the plate leading edge. This profile is compared with experimental curve fits in Fig. 11. The experimental curve fit is given as,

$$
u^{+}=\left\{\begin{aligned}
y^{+}: & y^{+}<10 \\
\frac{1}{0.41} \ln \left(y^{+}\right)+5: & y^{+} \geq 10
\end{aligned}\right.
$$

The output adapted CFD results approach the experimental curve fits for small $y^{+}$and large $y^{+}$until the edge of the boundary layer at $y^{+}=1000$. The comparison of CFD to the experimental curve fits is extremely favorable for the output-adaptive technique for these flow conditions.

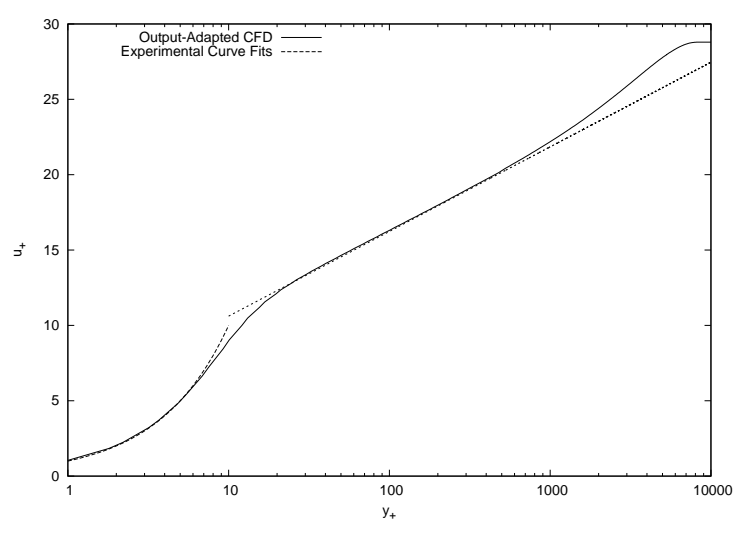

Figure 11. Flat-plate turbulent velocity profile. 


\section{Shock-Boundary Layer Interaction}

The shock-boundary layer experiment is described in Humble, Scarano, and van Oudheusden. ${ }^{42}$ This case is selected due to its inclusion in the AIAA Supersonic Shock-Boundary Layer Interaction (SBLI) Workshop. The computational domain is shown in Fig. 12, which consists of an extruded 2D converging-diverging nozzle exiting into the test section containing a wedge set at 8 deg incidence to the tunnel center line. The Reynolds number is 5,683 per $\mathrm{cm}$. The total to freestream pressure ratio is 11.56 and the total to freestream temperature ratio is 2.01. A total pressure and total temperature boundary condition ${ }^{37}$ is applied to the inlet face upstream of the wind tunnel throat. These total conditions are intended to result in an isentropic expansion to the Mach 2.25 test condition. This Mach number is combined with a stagnation pressure of 50.5 $\mathrm{kPa}$ and stagnation temperature of $295 \mathrm{~K}$ to dimensionalize the velocity for comparison with Particle Image Velocimetry (PIV) measurements. No slip boundary conditions are applied to the walls of the tunnel and the wedge shock generator. The outflow boundary condition is extrapolated from the interior of the domain. Drag on the floor of the tunnel test section is used as the output to drive adaptation. The problem was executed on 256 processors. The physics of shock-boundary layer interaction is inherently time-dependent. However, a steady-state simulation was employed in this study to obtain mean values.

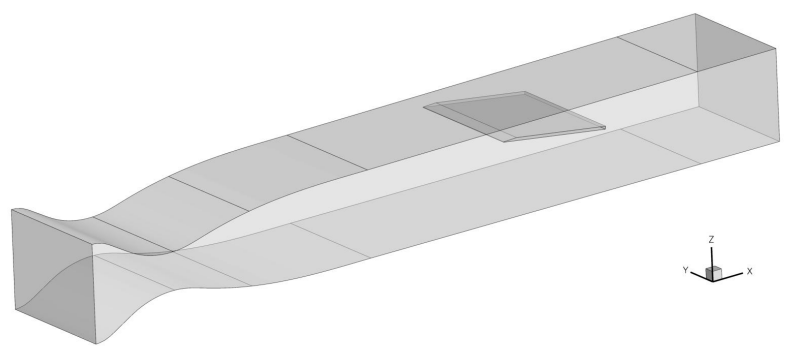

Figure 12. Shock-boundary layer test geometry.

The original grid and adapted grid velocity fields along the center plane of the tunnel are shown in Fig. 13. The $u$-velocity is given in units of meters per second. The shock and expansion structures are better defined for the adapted grid. The wedge shock generator wake is propagated a longer distance down stream. The shock-boundary layer interaction zone on the lower wall of the adapted grid has a larger region of reversed flow. The shock reflected off the lower boundary layer has increased in strength and is more clearly defined.

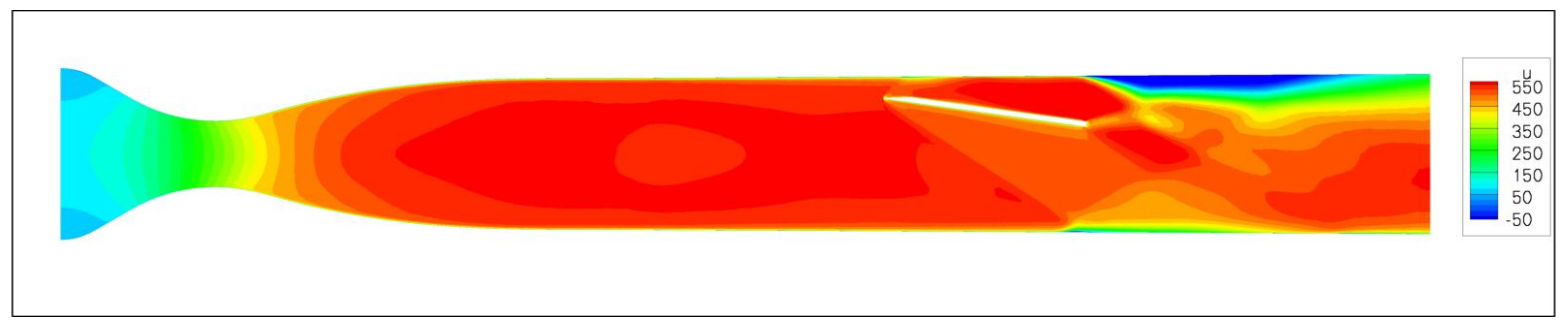

(a) Initial grid.

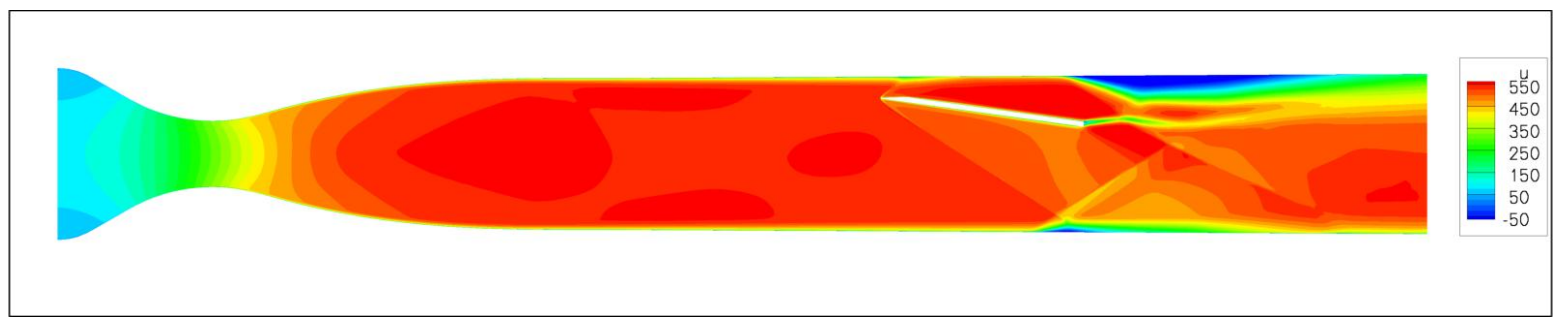

(b) Output adapted grid.

Figure 13. Velocity for the center plane $(\mathrm{m} / \mathrm{s})$.

The intersection of the original and adapted tetrahedral volume grids with the center plane of the wind tunnel are shown in Fig. 14. The original grid was generated with VGrid $^{38}$ and has an initial cell height of 
$0.005 \mathrm{~cm}$ with a growth rate of 1.15 and secondary rate acceleration of 0.08 . The boundary layer grid was frozen within $0.3 \mathrm{~cm}$ of the no slip boundary conditions. The initial grid has 766,384 nodes and the adapted grid has 1,301,836 nodes. The outer portions of the incoming boundary layers on the floor and roof of the test section have been refined. The bow shock of the wedge shock generator is tightly refined to propagate the shock to the shock-boundary layer interaction zone. The boundary layer above and below the wedge shock generator are better resolved. The shock reflected off the floor is also refined with the expansion off the the rear of the wedge. The adapted floor boundary layer grid is more isotropic at and down stream of the shock interaction zone due to increased stream wise Mach variation.

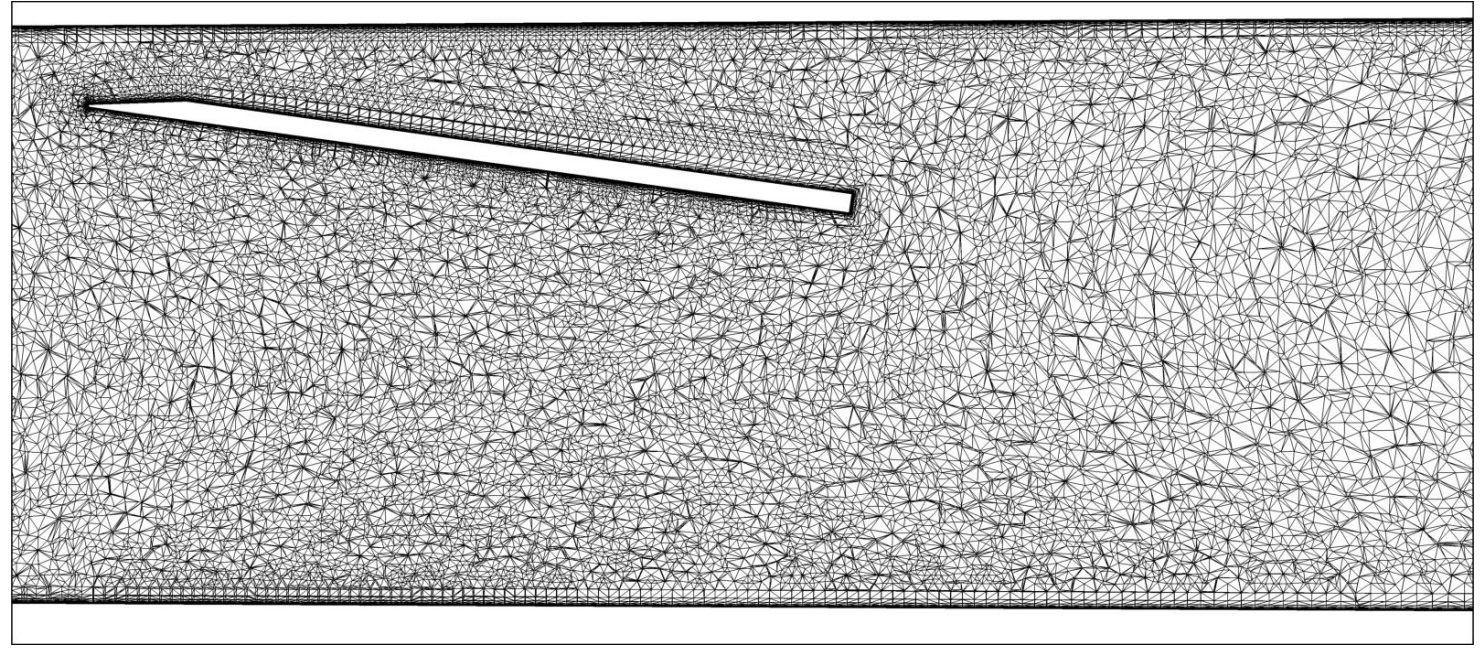

(a) Initial grid.

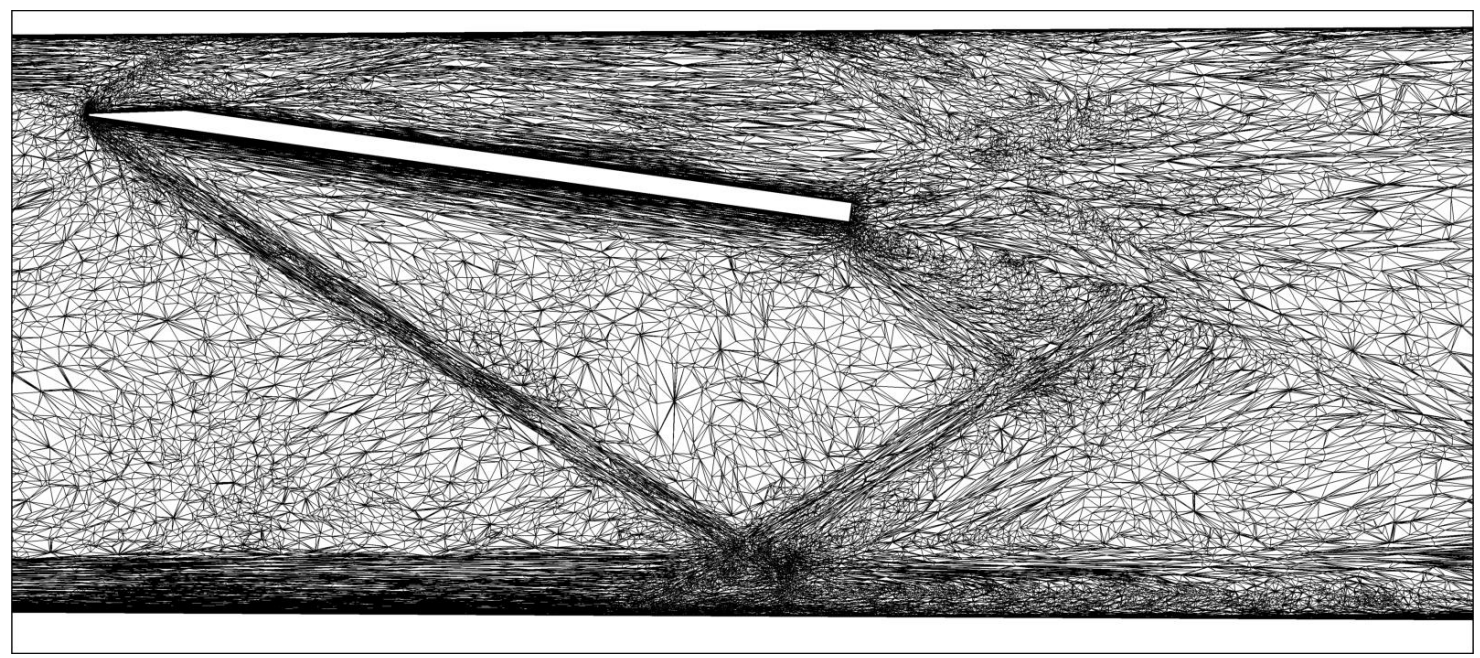

(b) Output adapted grid.

Figure 14. Detail of the intersection of the volume grid and center plane.

The adapted grid velocity and experimental PIV data are shown in Fig. 15. The $u$-velocity is given in units of meters per second. The PIV measurements are not available in the upper left of the figure. The incident boundary layer is slightly thicker for the PIV measurement. The adapted CFD predicts a larger region of reversed flow. The CFD incident shock location is slightly forward of the PIV measurement. This may be due to the steady-state approximation.

The adapted grid CFD and PIV data are extracted at various $x$ locations near the shock boundary layer interaction zone, see Fig. 16. The $x=260$ and 280 stations are upstream of the boundary layer thickening due to the shock interaction. The boundary layer thickens at $x=300$ and 310 with CFD predicting the reversed flow region to extend further upstream of the shock. The largest difference between the CFD prediction and the PIV measurement is at and down stream of $x=320$, where the CFD predicts higher velocity than the PIV measurement. 


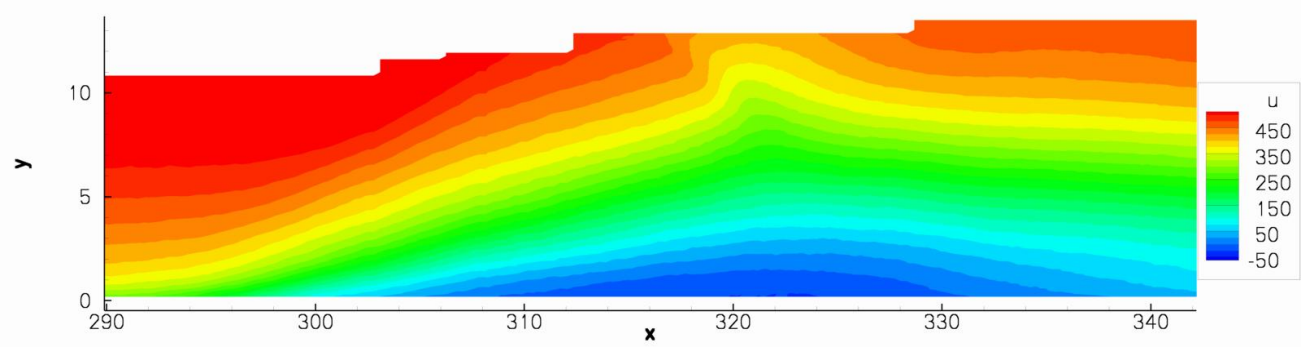

(a) Experimental time-averaged PIV $u$-velocity field.

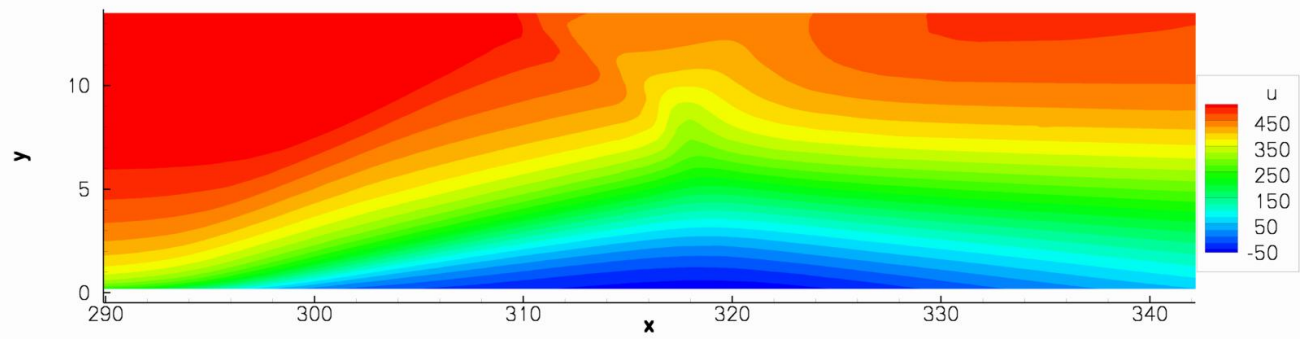

(b) Final adapted Steady-state CFD $u$-velocity field.

Figure 15. Detail of the shock-boundary layer interaction zone $(\mathrm{m} / \mathrm{s})$.

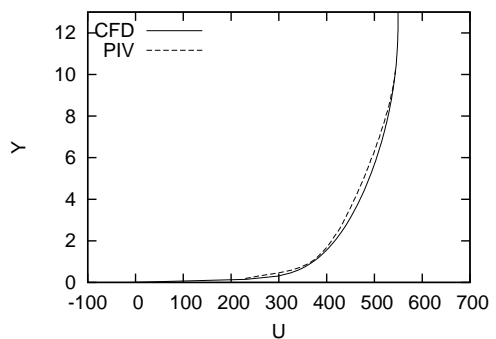

(a) $x=260$.

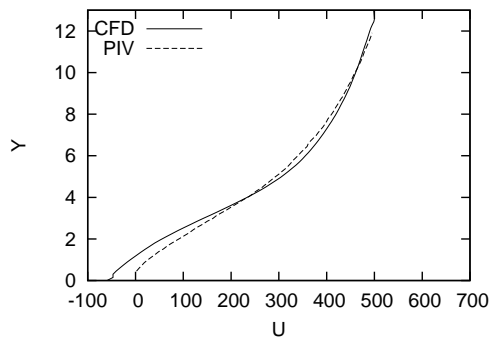

(d) $x=310$.

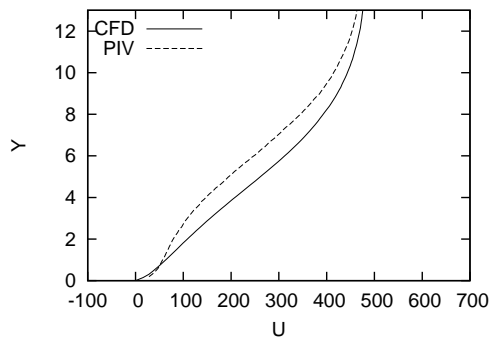

(g) $x=340$.

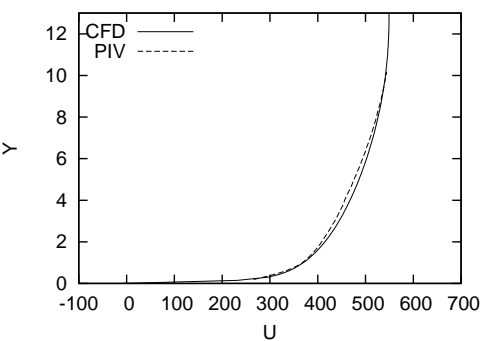

(b) $x=280$.

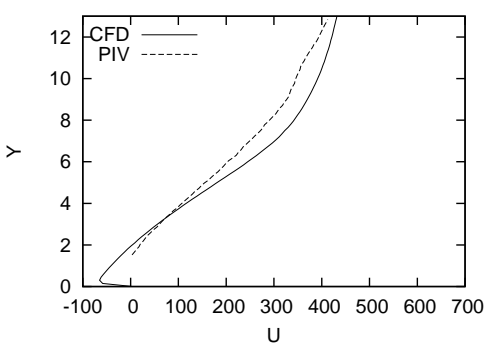

(e) $x=320$.

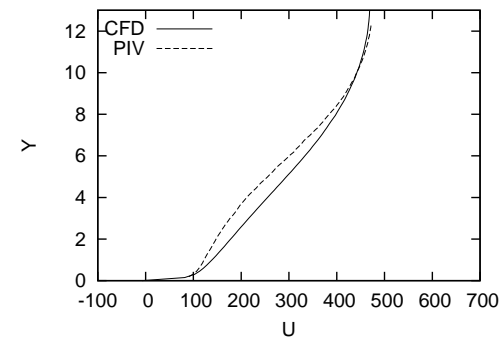

(h) $x=360$.

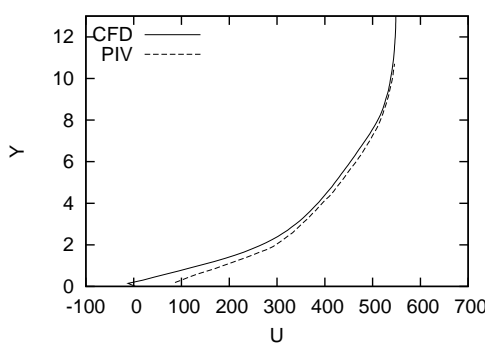

(c) $x=300$.

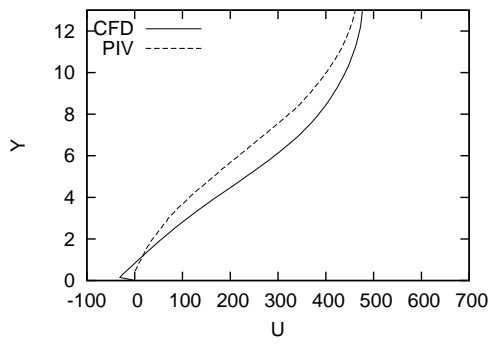

(f) $x=330$.

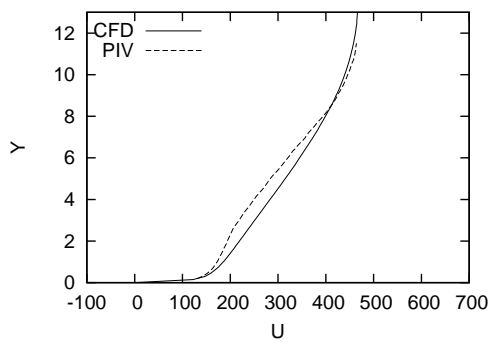

(i) $x=380$.

Figure 16. Velocity profiles at various stations for shock-boundary layer interaction zone $(\mathrm{m} / \mathrm{s})$. 


\section{Conclusions}

Grid adaptation is driven by estimated discretization error in drag and pressure integral output functions. To enable application to high $\mathcal{O}\left(10^{7}\right)$ Reynolds number turbulent flows a hybrid approach is utilized that freezes the near-wall portion of boundary layer grids and adapts the grid away from the no slip boundaries. Supersonic nozzle plume, turbulent flat plate, and shock-boundary layer interaction examples are presented with comparisons to experimental measurements of pressure and velocity. The conditions of the turbulent flat plate matched the grid and Reynolds number at the location of the boundary layer profile of the plume case and verified that the adaptive approach provides the expected boundary layer velocity profile for subsonic flow. Adapted grids are produced that resolve off-body features in locations that are not known a priori. These features include shocks, expansions, boundary layers, and shear layers. The grids are anisotropically adapted to each of these features that has a significant impact on the specified output. The regions where these features interact lose a preferred anisotropic direction so they are refined isotropically. The hybrid approach is not applicable to problems with under-resolved initial boundary layer grids, but is a powerful technique for problems with important off-body anisotropic features.

\section{References}

\footnotetext{
${ }^{1}$ Mavriplis, D. J., Vassberg, J. C., Tinoco, E. N., Mani, M., Brodersen, O. P., Eisfeld, B., Wahls, R. A., Morrison, J. H., Zickuhr, T., Levy, D., and Murayama, M., "Grid Quality and Resolution Issues from the Drag Prediction Workshop Series," AIAA Paper 2008-930, 2008.

${ }^{2}$ Mavriplis, D. J., "Grid Resolution Study of a Drag Prediction Workshop Configuration Using the NSU3D Unstructured Mesh Solver," AIAA Paper 2005-4729, 2005.

${ }^{3}$ Chaffin, M. S. and Pirzadeh, S., "Unstructured Navier-Stokes High-Lift Computations on a Trapezoidal Wing," AIAA Paper 2005-5084, 2005.

${ }^{4}$ Murman, S. M., Aftosmis, M. J., and Nemec, M., "Automated Parameter Studies Using a Cartesian Method," AIAA Paper 2004-5076, 2004.

${ }^{5}$ Nemec, M. and Aftosmis, M. J., "Aerodynamic Shape Optimization Using a Cartesian Adjoint Method and CAD Geometry," AIAA Paper 2006-3456, 2006.

${ }^{6}$ Venditti, D. A. and Darmofal, D. L., "Anisotropic Grid Adaptation for Functional Outputs: Application to TwoDimensional Viscous Flows," Journal of Computational Physics, Vol. 187, 2003, pp. 22-46.

${ }^{7}$ Dompierre, J., Vallet, M.-G., Bourgault, Y., Fortin, M., and Habashi, W. G., "Anisotropic mesh adaptation: towards user-independent, mesh-independent and solver-independent CFD. Part III. Unstructured grids," International Journal for Numerical Methods in Fluids, Vol. 39, No. 8, 2002, pp. 675-702.

${ }^{8}$ Fidkowski, K. J. and Darmofal, D. L., "A Triangular Cut-Cell Adaptive Method for High-Order Discretizations of the Compressible Navier-Stokes Equations," Journal of Computational Physics, Vol. 225, No. 2, Aug. 2007, pp. $1653-1672$.

${ }^{9}$ Wintzer, M., Nemec, M., and Aftosmis, M. J., "Adjoint-Based Adaptive Mesh Refinement for Sonic Boom Prediction," AIAA Paper 2008-6593, 2008.

${ }^{10}$ Peraire, J., Peirò, J., and Morgan, K., "Adaptive Remeshing for Three-Dimensional Compressible Flow Computations," Journal of Computational Physics, Vol. 103, No. 2, 1992, pp. 269-285.

${ }^{11}$ Baker, T. J., "Mesh Adaptation Strategies for Problems in Fluid Dynamics," Finite Elements in Analysis and Design, Vol. 25, No. 3-4, 1997, pp. 243-273.

${ }^{12}$ Aftosmis, M. J. and Berger, M. J., "Multilevel Error Estimation and Adaptive h-Refinement for Cartesian Meshes with Embedded Boundaries," AIAA Paper 2002-0863, 2002.

${ }^{13}$ Alauzet, F. and Loseille, A., "High-order sonic boom modeling based on adaptive methods," Journal of Computational Physics, Vol. 229, No. 3, 2009, pp. 561-593.

${ }^{14}$ Rannacher, R., "Adaptive Galerkin Finite Element Methods for Partial Differential Equations," Journal of Computational and Applied Mathematics, Vol. 128, 2001, pp. 205-233.

${ }^{15}$ Pierce, N. A. and Giles, M. B., "Adjoint Recovery of Superconvergent Functionals from PDE Approximations," SIAM Review, Vol. 42, No. 2, 2000, pp. 247-264.

${ }^{16}$ Venditti, D. A., Grid Adaptation for Functional Outputs of Compressible Flow Simulations, Ph.D. thesis, Massachusetts Institute of Technology, 2002.

${ }^{17}$ Fidkowski, K. J. and Darmofal, D. L., "Output Error Estimation and Adaptation in Computational Fluid Dynamics: Overview and Recent Results," AIAA Paper 2009-1303, 2009.

${ }^{18}$ Park, M. A., "Three-Dimensional Turbulent RANS Adjoint-Based Error Correction," AIAA Paper 2003-3849, 2003.

${ }^{19}$ Lee-Rausch, E. M., Park, M. A., Jones, W. T., Hammond, D. P., and Nielsen, E. J., "Application of a Parallel AdjointBased Error Estimation and Anisotropic Grid Adaptation for Three-Dimensional Aerospace Configurations," AIAA Paper 2005-4842, 2005.

${ }^{20} \mathrm{Li}$, X., Shephard, M. S., and Beall, M. W., "Accounting for curved domains in mesh adaptation," International Journal for Numerical Methods in Engineering, Vol. 58, No. 1, 2000, pp. 247-276.

${ }^{21} \mathrm{Li}$, X., Mesh Modification Procedures for General 3-D Non-Manifold Domains, Ph.D. thesis, Rensselaer Polytecnic Institute, 2003.
} 
${ }^{22}$ Karamete, B. K., Beall, M. W., and Shephard, M. S., "Triangulation of arbitrary polyhedra to support automatic mesh generators," International Journal for Numerical Methods in Engineering, Vol. 49, No. 12, 2000, pp. 167-191.

${ }^{23}$ Anderson, W. K. and Bonhaus, D. L., "An Implicit Upwind Algorithm for Computing Turbulent Flows on Unstructured Grids," Computers and Fluids, Vol. 23, No. 1, 1994, pp. 1-22.

${ }^{24}$ Anderson, W. K., Rausch, R. D., and Bonhaus, D. L., "Implicit/Multigrid Algorithm for Incompressible Turbulent Flows on Unstructured Grids," Journal of Computational Physics, Vol. 128, No. 2, 1996, pp. 391-408.

${ }^{25}$ Spalart, P. R. and Allmaras, S. R., "One-Equation Turbulence Model for Aerodynamic Flows," AIAA Paper 92-439, 1992.

${ }^{26}$ Spalart, P. R. and Allmaras, S. R., "A One-Equation Turbulence Model for Aerodynamic Flows," La Recherche Aerospatiale, , No. 1, 1994, pp. 5-21.

${ }^{27}$ Nielsen, E. J. and Anderson, W. K., "Recent Improvements in Aerodynamic Design Optimization on Unstructured Meshes," AIAA Journal, Vol. 40, No. 6, 2002, pp. 1155-1163, See also AIAA Paper 2001-596.

${ }^{28}$ Nielsen, E. J., Aerodynamic Design Sensitivities on an Unstructured Mesh Using the Navier-Stokes Equations and a Discrete Adjoint Formulation, Ph.D. thesis, Virginia Polytechnic Institute and State University, 1998.

${ }^{29}$ Giles, M., Duta, M., Müller, J.-D., and Pierce, N., "Algorithm Developments for Discrete Adjoint Methods," AIAA Journal, Vol. 41, No. 2, 2003, pp. 198-205, See also AIAA Paper 2001-2596.

${ }^{30}$ Nielsen, E. J., Lu, J., Park, M. A., and Darmofal, D. L., "An Implicit, Exact Dual Adjoint Solution Method for Turbulent Flows on Unstructured Grids," Computers and Fluids, Vol. 33, No. 9, 2004, pp. 1131-1155, See also AIAA Paper $2003-272$.

${ }^{31}$ Park, M. A., Anisotropic Output-Based Adaptation with Tetrahedral Cut Cells for Compressible Flows, Ph.D. thesis, Massachusetts Institute of Technology, Sept. 2008.

${ }^{32}$ Habashi, W. G., Dompierre, J., Bourgault, Y., Ait-Ali-Yahia, D., Fortin, M., and Vallet, M.-G., "Anisotropic mesh adaptation: towards user-independent, mesh-independent and solver-independent CFD. Part I: general principles," International Journal for Numerical Methods in Fluids, Vol. 32, No. 6, 2000, pp. 725-744.

${ }^{33}$ Castro-Diáz, M. J., Hecht, F., Mohammadi, B., and Pironneau, O., "Anisotropic Unstructured Mesh Adaptation for Flow Simulations," International Journal for Numerical Methods in Fluids, Vol. 25, No. 4, 1997, pp. $475-491$.

${ }^{34}$ Frey, P. J. and Alauzet, F., "Anisotropic mesh adaptation for CFD computations," Computer Methods in Applied Mechanics and Engineering, , No. 194, Nov. 2005, pp. 5068-5082.

${ }^{35}$ Park, M. A. and Darmofal, D., "Parallel Anisotropic Tetrahedral Adaptation," AIAA Paper 2008-917, 2008.

${ }^{36}$ Putnam, L. E. and Capone, F. J., Experimental Determination of Equivalent Solid Bodies to Represent Jets Exhausting into a Mach 2.20 External Stream, NASA-TN-D-5553, Dec. 1969.

${ }^{37}$ Carlson, J.-R., "Inflow/Outflow Boundary Conditions with Application to FUN3D," NASA TM, NASA Langley Research Center, 2010, to appear.

${ }^{38}$ Pirzadeh, S. Z., "Three-Dimensional Unstructured Viscous Grids by the Advancing-Layers Method," AIAA Journal, Vol. 34, No. 1, Jan. 1996, pp. 43-49.

${ }^{39}$ Castner, R. S., "Analysis of Plume Effects on Sonic Boom Signature for Isolated Nozzle Configurations," AIAA Paper 2008-3729, 2008.

${ }^{40}$ Park, M. A. and Darmofal, D., "Output-Adaptive Tetrahedral Cut-Cell Validation for Sonic Boom Prediction," AIAA Paper 2008-6594, 2008.

${ }^{41}$ Andren, J. and Park, M. A., "Output Based Grid Adaptation for Viscous Flow," 18th International Meshing Roundtable Research Notes, Sandia National Lab, Oct. 2009, pp. 1-5.

${ }^{42}$ Humble, R. A., Scarano, F., and van Oudheusden, B., "Experimental Study of an Incident Shock Wave/Turbulent Boundary Layer Interaction Using PIV," AIAA Paper 2006-3361, 2006. 